\title{
The Role of ACE-Inhibitors in Patients with Coronary Artery Disease
}

\author{
Bertram Pitt \\ University of Michigan, School of Medicine, Ann Arbor, \\ Michigan
}

\begin{abstract}
$\boldsymbol{A C E}$-inhibitors have been shown to be effective in reducing morbidity and mortality in patients with heart failure due to systolic left ventricular dysfunction, post myocardial infarction, in patients with hypertension, and in patients with renal disease associated with diabetes mellitus. The heart outcome prevention trial (HOPE) extended these indications by showing that patients with vascular disease without a history of heart failure and those with diabetes mellitus with an additional risk factor had a significant reduction in death, nonfatal myocardial infarction, nonfatal stroke, need for revascularization, and new onset of diabetes mellitus [1]. These findings are supported by a wide variety of pre-clinical and clinical studies showing that ACE-inhibitors reduce oxidative stress, improve nitric oxide availability, endothelial function, activation of adhesion molecules and cytokines, transport and oxidation of LDL cholesterol into macrophages, composition of the atherosclerotic plaque, as well as preventing myocardial and perivascular fibrosis and ventricular hypertrophy [2-5].
\end{abstract}

Given the accumulating data showing the benefit of ACE-inhibitors in reducing morbidity and mortality in patients with coronary artery disease what can we expect to learn from further trials in this area such as EUROPA.

EUROPA has much to teach us and its results will have important implications for future practice. One important question to be answered by this study is whether the results of HOPE are specific for ramipril or are due to a "class effect" of ACE-inhibitors. As pointed out above there is considerable pre-clinical and clinical data with a variety of ACE-inhibitors elucidating the mechanisms by which an ACE-inhibitor might reduce ischemic events. While a specific effect of ramipril cannot be ruled out it is likely that perindopril (EUROPA) will also be effective in reducing ischemic events. While a class effect is likely it is possible that one or another ACE-inhibitor will be more effective then another, possibly related to tissue selectivity, duration of action, and perhaps most importantly dosing strategy [6]. As more ACE-inhibitors become available generically, and therefore at relatively low cost it will be important to have assurance that the beneficial effects seen in the HOPE trial can be achieved with other ACE-inhibitors. However, even if EUROPA confirms the findings of the HOPE trial clinicians may still face a dilemma in extrapolating the results of these trials to other ACE-inhibitors, since the dose of an ACE-inhibitor appears to be critical in its ability to reduce ischemic events. For example, the results of the Secure study using ramipril $2.5 \mathrm{mg}$ or $10 \mathrm{mg}$ examining their effect on the progression on atherosclerosis, as reflected by carotid intima media thickness, show that $10 \mathrm{mg}$ daily but not $2.5 \mathrm{mg}$ daily was effective in reducing the progression of atherosclerosis [7]. Therefore, it may be hazardous to extrapolate from the results of EUROPA, even if this study is positive to other ACE-inhibitors for which we have no clinical evidence of effectiveness at a given dose in this indication. It would, I believe, be hazardous to conclude that two ACE-inhibitors which reduce blood pressure to an equal degree at given doses will be equivalent in reducing ischemic events to the same degree. With these considerations in mind it would also be hazardous to reject the findings in HOPE even if EUROPA is negative. Not only are there differences in the individual ACE-inhibitors, and their dose, but there are also important differences in patient inclusions in these trials. For example in EUROPA there is a far broader and possibly lower risk population of patients with coronary artery disease then in HOPE.

Aside from the question as to class effect what else can we hope to learn from EUROPA. EUROPA in my opinion may be particularly important in answering the question as to whether the mechanism of ACE-inhibitors in reducing nonfatal myocardial infarction, as seen in HOPE, is related to an effect of an ACE-inhibitor on the progression of coronary atherosclerosis, plaque rupture, or thrombosis after plaque rupture. While ACE-inhibitors have been shown to prevent experimental atherosclerosis their role in preventing the progression of coronary atherosclerosis in man is uncertain. The angiographic sub-study of the QUIET trial of quinapril $20 \mathrm{mg}$ daily failed to show a significant effect of quinapril on the progression of coronary atherosclerosis [8]. A retrospective analysis of the QUIET trial has however suggested that there was a significant effect of quinapril in preventing coronary atherosclerosis in patients with an elevated LDL-cholesterol above the median in that trial, $126 \mathrm{mg}$ dl. An angiographic study of enalapril (Scat) also failed to demonstrate a significant effect on the progression of coronary atherosclerosis 
[9]. The secure study showed a significant effect of ramipril $10 \mathrm{mg}$ daily on the progression of carotid intima media thickness however the Part 2 study with ramipril failed to demonstrate a significant effect on carotid intimal thickness [10]. Differences in methodology and dose have been suggested to account for the discrepant results of ramipril on carotid intima media thickness. Regardless, at the moment we do not have an adequate explanation as to why ramipril $10 \mathrm{mg}$ reduced the incidence of nonfatal infarction and ischemic events in HOPE. One might postulate that ramipril was effective in reducing ischemic events not by preventing the progression of coronary atherosclerosis but by preventing plaque rupture and or thrombosis after plaque rupture. Angiotensin II and the converting enzyme have been localized in the shoulder region of the atherosclerotic plaque $[11,12]$ and $\mathrm{ACE}$ inhibitors shown to decrease metaloproteinase activity in the plaque and increase collagen content, thereby improving plaque stability. Angiotensin II by stimulating NADH/NADPH oxidases in the vascular wall [13] and oxygen free radical release have been shown to stimulate various signaling pathways including NFKB and AP1, which have been associated with a decrease nitric oxide availability, an increase in tissue factor, and plasminogin activator inhibitor levels which would increase thrombosis, and decrease fibrinolysis after plaque rupture. In addition to its effects on fibrinolysis plasminogen activator inhibitor may also contribute to plaque instability. Possibly several processes such as progression of coronary atherosclerosis, plaque instability, and thrombosis play an important role in the effect of ACE-inhibitors in reducing ischemic events. The sub-studies of EUROPA examining the effect of perindopril on coronary atherosclerosis by intravascular ultrasound, coagulation factors, and plasminogin activator inhibitor levels should help to clarify the relative importance of these mechanisms. While it can be argued that the exact mechanism by which an ACEinhibitor reduces the incidence of nonfatal myocardial infarction and ischemic events is less important then the fact that ischemic events are reduced an understanding of the mechanism by which ischemic events are reduced will be useful in designing future strategies. For example, should an ACE-inhibitor be shown to be effective in preventing new coronary artery lesion formation, such as has been demonstrated with a statin, agents that further improve plaque stability and/or agents that decrease thrombosis after plaque rupture may be important. Conversely, if ACE-inhibitors prevent plaque rupture without effecting the progression of atherosclerosis agents such as a statin which have been clearly shown to prevent new coronary lesion formation and the progression of atherosclerosis may be important adjunctive strategies.

Clearly, the results of EUROPA will have important implications for future practice in regard to the question of class effectiveness, extrapolation of the results of HOPE to a broader group of patients with coronary artery disease and possibly patients with lower risk profiles, and an understanding of the mechanisms by which the beneficial effects of ACE-inhibitors are achieved. EUROPA is however only a waypoint in our understanding of the role of the renin angiotensin system in ischemic heart disease. Other trials of ACEinhibitors such as PEACE with trandolapril and new trials comparing ACE-inhibitors to angiotensin receptor blocking agents and their combination are underway as well as the new trials exploring the effects of ACE-inhibitors to prevent diabetes mellitus. There is also emerging data that aldosterone receptor blocking agents may have an important effect in reducing ischemic events in view of their effectiveness in restoring endothelial function and reducing oxygen free radical formation in experimental hyperlipidemic rabbits. Further studies exploring the synergestic or additive effects of ACE-inhibitors and statins, ACEinhibitors, and antithrombotic or anti-platelet strategies, as well as anti-inflammatory strategies are clearly needed. Although the epidemic of ischemic heart disease continues the demonstration of the effectiveness of statins, ACE-inhibitors, anti-thrombotic, and antiplatelet strategies make me hopeful although that the epidemic is not eradicated it is being contained. Only through further well-designed pre-clinical studies and large-scale randomized trials such as EUROPA can we hope to claim victory.

\section{References}

1. The Heart Outcomes Prevention Evaluation Study Investigators. Effects of an angiotensin-converting-enzyme inhibitor, ramipril, on cardiovascular events in high-risk patients. N Engl J Med 2000;342:145-53.

2. Keidar S, Kaplan M, Aviram M. Angiotensin II-modified LDL is taken up by macrophages via the scavenger receptor, leading to cellular cholesterol accumulation. Aterioscler Thromb Vasc Biol 1996;16:97-05.

3. Mancini GBJ, Henry GC, Macaya C, et al. Angiotensinconverting enzyme inhibition with quinapril improves endothelial vasomotor dysfunction in patients with coronary artery disease: The TREND (Trial on Reversing ENdothelial Dysfunction) Study. Circulation 1996;94:258-65.

4. Pepine CJ, Pitt B, Bertrand ME, Timmis A. Emerging role of ACE inhibitors in vascular protection. J Myocard Ischemia 1995;7(suppl 1):50-58.

5. Lonn EM, Yusuf S, Jha P, et al. Emerging role of angiotensin-converting enzyme inhibitors in cardiac and vascular protection. Circulation 1994;90:2056-69.

6. Furberg CD, Pitt B. Are all angiotensin-converting enzyme inhibitors interchangeable. J Am Coll Cardiol 2001; 37:1456-60.

7. Lonn EM, Yusuf S, Dzavik V, et al. Effects of ramipril and vitamin $\mathrm{E}$ on atherosclerosis: The study to evaluate carotid ultrasound changes in patients treated with ramipril and vitamin E (SECURE). Circulation 2001;103:919-25.

8. Texter M, Lees RS, Pitt B, et al. The quniapril ischemic event trial (QUIET) design and methods: Evaluation of chronic ACE inhibitor therapy after coronary artery intervention. Cardiovascular Drugs Ther 1993;7:273-82. 
9. Teo K, Burton J, Buller C, et al. Long-term effects of cholesterol lowering and angiotensin-converting enzyme inhibition on coronary atherosclerosis. Circulation 2000;102:1748-54.

10. MacMahon S, Sharpe N, Gamble G, et al. Randomized, placebo-controlled trial of th eangiotensin-converting enzyme inhibitor, ramipril, in patients with coronary or other occlusive arterial disease. J Am Coll Cardiol 2000;36:438-43.

11. Ohishi M, Ueda M, Rakugi H, Naruko T, Kojima A, Okamura A, et al. Relative localization of angiotensinconverting enzyme, chymase and angiotensin II in human coronary atherosclerotic lesions. J Hypertens 1999;17:54753.

12. Potter DD, Sobey CG, Tompkins PK, Rossen JD, Heistad DD. Evidence that macrophages in atherosclerotic lesions contain angiotensin II. Circulation 1998;98:800-07.

13. Warnholtz A, Nickenig G, Schulz E, Macharzina R, Brasen $\mathrm{JH}$, Skatchkov M, et al. Increased NADH-oxidase-mediated superoxide production in the early stages of atherosclerosis: Evidence for involvement of the renin-angiotensin system. Circulation 1999;15:2027-33. 